\title{
Stellar Alignment of the High Resolution Doppler Imager
}

\author{
Alan R. Marshall, ${ }^{*}$ David A. Gell, ${ }^{\dagger}$ Jeng-Hwa Yee, ${ }^{\ddagger}$ David A. Ortland, ${ }^{\dagger}$ Mark D. Burrage, ${ }^{\S}$ Wilbert R. Skinner ${ }^{\mathbb{I}}$ \\ and \\ Paul B. Hays** \\ University of Michigan, Ann Arbor, Michigan 48109-2143
}

\begin{abstract}
A method is described for determining the angular misalignment between the High Resolution Doppler Imager instrument and its spacecraft, the Upper Atmospheric Research Satellite, using observations of stars by the instrument. Roll, pitch, and yaw misalignments have been measured to within 0.0025 deg $(1 \sigma)$, which is an order of magnitude better than the largest tolerable alignment error. The misalignment results are validated using observations of the nighttime 5577- $\AA \mathrm{O}\left({ }^{1} S\right)$ green line emission. In addition, the long-term behavior of the derived alignment offsets, which shows a clear dependence of the alignment on the solar $\beta$ angle, is presented.
\end{abstract}

\author{
Nomenclature \\ $p_{x} \quad=$ vector in the instrument's viewing direction expressed \\ in the $x$ coordinate frame \\ $T_{x / y} \quad=$ transformation matrix from the $y$ to the $x$ coordinate \\ frame \\ $=$ measured time of the star crossing, $\mathrm{s}$ \\ $=$ calculated star crossing time for the known star \\ position and spacecraft position and attitude, $s$ \\ $\begin{array}{ll}\eta_{0} & =\text { known angular misalignment vector, deg } \\ \eta_{1} & =\text { angular misalignment between the instrument and }\end{array}$ \\ spacecraft frame about the roll axis of the spacecraft, \\ deg \\ $\eta_{2} \quad=$ angular misalignment between the instrument and \\ spacecraft frame about the pitch axis of the spacecraft, \\ deg \\ $\eta_{3} \quad=$ angular misalignment between the instrument and \\ spacecraft frame about the yaw axis of the spacecraft, \\ deg \\ $\partial \eta / \partial t=$ derivative of the misalignment with respect to crossing \\ time expressed as a three-element column vector (in \\ roll, pitch, and yaw), deg/s
}

\section{Introduction}

$\mathbf{T}$ HE High Resolution Doppler Imager (HRDI) measures winds and other quantities in the stratosphere, mesosphere, and lower thermosphere by examining the absorption and emission lines of molecular oxygen. ${ }^{1,2}$ HRDI views small volumes of the atmospheric limb of the Earth using a two-axis gimbaled telescope. Winds along the instrument line of sight (LOS) are determined by measuring the Doppler shift of the observed lines. The meridional and zonal components of the winds are resolved by combining two approximately orthogonal LOS observations of the same volume in the atmosphere, one taken looking forward at an azimuth of $45 \mathrm{deg}$ to the spacecraft velocity vector, and the other taken approximately 9 min later looking backward at an azimuth of $135 \mathrm{deg}$. One of the chief goals of the HRDI project is to measure these winds to an accuracy of $5 \mathrm{~m} / \mathrm{s}$. Further, in order to make these measurements as useful as possible, their position also needs to be determined accurately.

Received Dec. 21, 1994; revision received April 18, 1995; accepted for publication April 18, 1995. Copyright (C) 1995 by the American Institute of Aeronautics and Astronautics, Inc. All rights reserved.

*Programmer Analyst, Space Physics Research Laboratory.

${ }^{\dagger}$ Senior Research Associate, Space Physics Research Laboratory.

¥ Space Physics Research Laboratory; currently Senior Research Scientist, Applied Physics Laboratory, Johns Hopkins University, Laurel, MD 207236099 .

${ }^{\S}$ Assistant Research Scientist, Space Physics Research Laboratory.

IAssociate Research Scientist, Space Physics Research Laboratory.

** Professor, Research Scientist, Space Physics Research Laboratory.
One complication in the method used by HRDI for measuring winds is that the Doppler shift in the observed lines is created by the total relative motion of the spacecraft and the viewed atmospheric region. This quantity includes not only the atmospheric motion relative to the Earth, but also the motion of the spacecraft relative to the Earth. The component of the spacecraft motion along the line of sight depends on three quantities: the spacecraft velocity relative to the Earth, the spacecraft orientation (attitude), and the orientation of the HRDI telescope relative to the spacecraft. The first two of these are well known, the spacecraft position and velocity are known to within $10 \mathrm{~m}$ and $0.5 \mathrm{~m} / \mathrm{s}$, and the spacecraft attitude can be determined to within better than $0.01 \mathrm{deg}(1 \sigma))^{3}$ Inaccuracies in the spacecraft velocity and attitude thus introduce very little error in the recovered winds (typically less than $1 \mathrm{~m} / \mathrm{s}$ ).

Because the satellite velocity is large compared to the wind being observed, the orientation of the telescope line of sight relative to the spacecraft velocity vector must be determined very accurately. To meet HRDI's goal of $2.5-\mathrm{m} / \mathrm{s}$ wind measurement error due to the spacecraft Doppler shift, the alignment of the telescope with respect to the spacecraft must be known to within $0.028 \mathrm{deg}$. The telescope alignment affects not only the accuracy of the derived winds, but also the determination of the measurement location. To meet the requirement of $1.5-\mathrm{km}$ error in tangent-point altitude, the alignment must be known to within $0.03 \mathrm{deg}$. While the alignment between the instrument and the spacecraft was measured accurately on the ground before launch, it is expected that the postlaunch alignment will be different because of the effects of gravity on the prelaunch measurement, launch-induced shifts, and thermal effects. With these small tolerances for knowledge of the instrument/spacecraft misalignments, a method for their measurement in space was necessary.

Attitude determination for spinning spacecraft ${ }^{4,5}$ and indeed for the UARS spacecraft itself ${ }^{6}$ has been done in the past by measuring the transit times of stars across one or more detectors' fields of view. A good entry to the literature is provided by Shuster et al. ${ }^{7}$ The adaptability of the HRDI instrument, whose sensitivity can be altered in orbit, makes it possible to view stars with HRDI and get sufficient signal-to-noise ratio to measure their transits accurately. Further, with its two-axis gimbaled telescope, the instrument can be programmed to actively "seek out" certain star crossings, making alignment measurement quicker and better than if the instrument field of view were fixed with respect to the spacecraft. Using measurements of stars' transit times and other available data, the HRDI-UARS misalignments can be calculated by several different methods. The analysis method outlined below is easily implemented and computationally simple, and yet provides more than sufficient accuracy for our purposes.

\section{Alignment Measurement Technique}

The in-orbit measurement of the HRDI-UARS alignment is performed in three steps: planning, data collection, and analysis. The 
planning step makes use of the predicted UARS orbit and the UARS Mission Star Catalog (which is an updated version of the SKYMAP catalog ${ }^{8}$ as used by the Landsat satellite) to determine available target stars. For each planned measurement period (typically half an orbit per week), the database is searched to find the brightest star that is both available for viewing (within the portion of the sky the instrument can view) and in a viewing direction that was not used to view prior target stars. For each target identified, the predicted viewing direction and time are used to develop an observation program. Up to 35 targets are used in the program, which controls the data collection step.

Data collection is performed using the HRDI photometer channel. A Schott Glass RG645 filter was used to prevent contamination by the $630-\mathrm{nm} \mathrm{O}\left({ }^{1} D\right)$ emission. The sensitivity of the detector vanished by $800 \mathrm{~nm}$, giving an effective bandpass of $150 \mathrm{~nm}$. For each target the telescope is positioned to view the star. Data are collected beginning $15 \mathrm{~s}$ prior to the predicted star crossing time, and collection continues for $30 \mathrm{~s}$. These data are part of the normal HRDI science data and are processed into dimensional quantities (times, telescope angles, and observed brightnesses) as part of the HRDI production software. The observed crossing time of a star is determined as the average of the times the observed brightness is larger than a threshold value, which is $10-20$ times the measured background level. Measurements that record multiple star crossings or no brightness above the threshold are discarded.

\section{Alignment Measurement Analysis}

The viewing direction of HRDI can be expressed as a vector $p$ with coordinates in the instrument frame. The viewing direction in the Earth-centered inertial (eci) frame is then given by

$$
p_{\text {eci }}=T_{\text {(eci/sc) }} T_{(\mathrm{sc} / \mathrm{inst})} \boldsymbol{p}_{\text {inst }}
$$

where $T_{x / y}$ is the rotation matrix that transforms a vector from the $y$ to the $x$ coordinate frame, and the subscripts sc, inst, and eci denote the spacecraft (UARS), instrument (HRDI), and Earth-centered inertial frames, respectively. The HRDI and UARS frames are illustrated in Fig. 1. Prior to launch, the alignment of the instrument frame to the spacecraft frame was measured as the misalignments between a series of coordinate frames:

$$
p_{\text {eci }}=T_{(\mathrm{eci} / \mathrm{sc})} T_{(\mathrm{sc} / \mathrm{obs})} T_{(\mathrm{obs} / \mathrm{inst})} \boldsymbol{p}_{\text {inst }}
$$

where the obs frame is the coordinate frame attached to the observatory structure and may be misaligned with the spacecraft attitude control system. The measurement method described hereafter estimates the elements of the rotation matrix

$$
T_{\text {(sc/inst) }}=T_{\text {(sc/obs) }} T_{\text {(obs/inst) }}
$$

The elements of the misalignment matrix $T_{\text {sc/inst }}$ are functions of the misalignments about the roll, pitch, and yaw axes of the spacecraft, $\eta_{1}, \eta_{2}$, and $\eta_{3}$, respectively:

$$
T_{\text {sc } / \text { inst }}=\left[\begin{array}{c}
\cos \eta_{2} \cos \eta_{3} \\
\cos \eta_{2} \sin \eta_{3}+\sin \eta_{1} \sin \eta_{2} \cos \eta_{3} \\
-\cos \eta_{1} \sin \eta_{2}
\end{array}\right.
$$

The present measurement technique makes use of the fact that when a star is centered in the field of view of the HRDI telescope, the viewing direction in the eci coordinate frame is known in addition to the spacecraft attitude, and thus any offset between the star's apparent position and its known location (from the star catalog) can be assumed to arise from misalignments between the HRDI instrument and the spacecraft frame. The search for stars is simplified by the orbital motion of the UARS spacecraft; the spacecraft velocity vector is in the direction of the roll axis, and the Earth is kept in the direction of the yaw axis as the spacecraft completes a full circle of pitch rotation each orbit. This pitch rotation causes an apparent

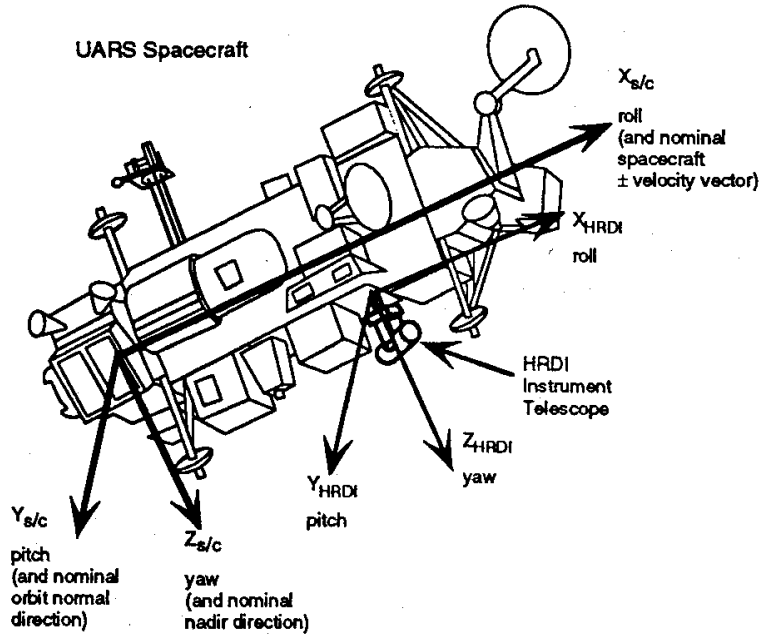

Fig. 1 HRDI and UARS coordinate frames.

circular motion of the stars about the pitch axis, $360 \mathrm{deg}$ per orbit (which takes about $90 \mathrm{~min}$ ), or about $4 \mathrm{deg}$ per minute. Since this motion is large compared with the HRDI telescope field of view ( 0.1 deg wide in elevation by 1.1 deg long in azimuth) for most stars, and since the misalignments are small, the telescope can simply be pointed slightly ahead of a target star, and data can be collected for a short time while the star transits the telescope field of view. A star in the roll-axis direction, for instance, will cross the HRDI telescope field of view (width) in about $1.5 \mathrm{~s}$, whereas a star in the roll-pitch plane, $10 \mathrm{deg}$ from the pitch axis, will take about $8.5 \mathrm{~s}$ to cross. The time at which the star is centered in the field of view may be expressed as a function of the misalignment,

$$
t-t_{0}=f(\eta)
$$

where $t$ is the measured time of the star crossing (when the star is centered in the field of view), and $t_{0}$ is the crossing time calculated for the known star position and spacecraft position and attitude. This can be linearized to obtain a measurement model,

$$
\eta-\eta_{0}=\frac{\partial \eta}{\partial t}\left(t-t_{0}\right)
$$

where the derivative $\partial \eta / \partial t$ is a three-element column vector (in roll, pitch, and yaw) which depends on the viewing direction. This vector is numerically evaluated using the UARS orbital ephemeris by evaluating the star crossing times, assuming first a small positive misalignment and then a small negative misalignment about each axis. The two calculated star crossing times are then used to estimate the derivative.

The misalignment measurement technique may be clarified by examining the simplest cases, using Fig. 2a. When the telescope

is viewing almost directly forward, along the spacecraft roll axis, the error in crossing time is due almost entirely to misalignments about the pitch axis. (Imagine wrapping Fig. 2a around you to cover one hemisphere, then rotating it about the roll axis slightly; this causes very little change in the positions of stars near the roll axis, and thus the crossing times of stars near azimuth 0 deg are not very sensitive to roll-axis misalignments. Rotation about the roll axis has a more pronounced effect on the apparent position of stars near the pitch axis, though; thus, these stars are sensitive to rollaxis misalignments.) Figure $2 b$ shows the three elements of the derivative $\partial \eta / \partial t$ as a function of azimuth angle for an elevation 

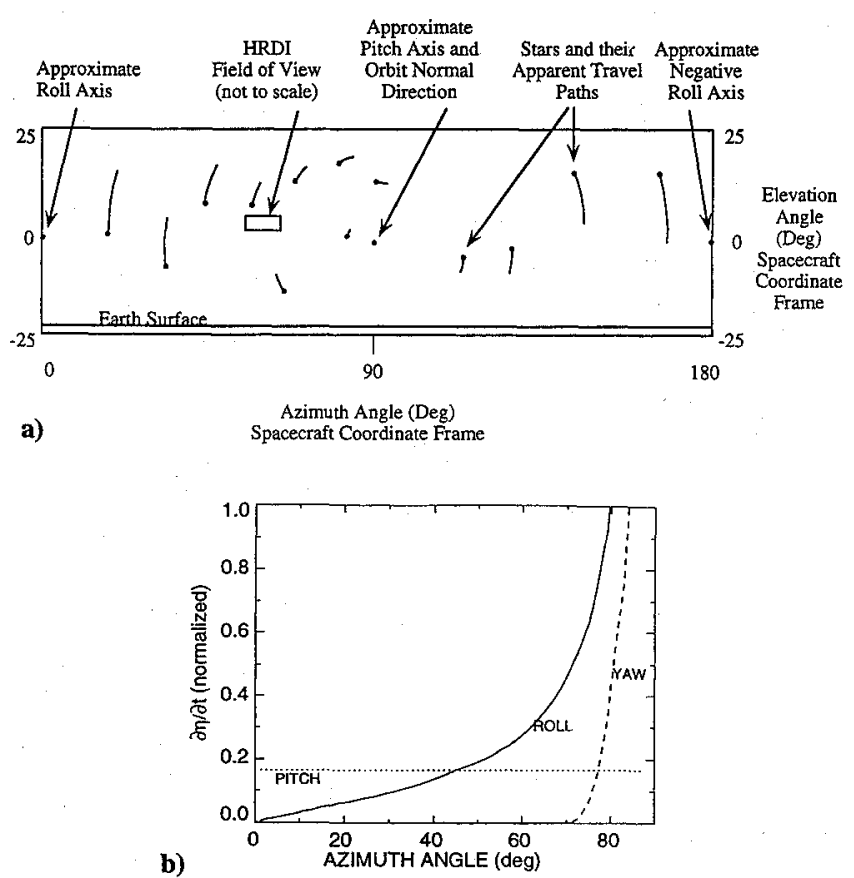

Fig. 2 a) Misalignment measurement conditions. b) $\partial \eta / \partial t$ for roll, pitch, and yaw as a function of azimuth angle (for elevation angle -0 deg).

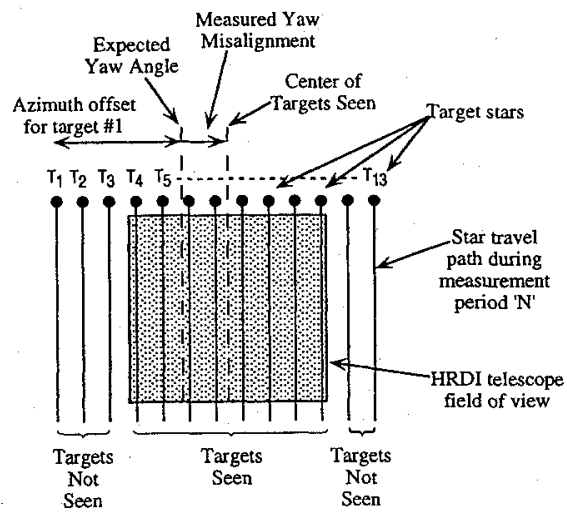

Fig. 3 Raster-scan measurement scheme for yaw misalignment.

angle of $-10 \mathrm{deg}$ (the values here are normalized so that the largest plotted value is 1.0). Stars are selected as targets that are at a variety of azimuth and elevation angles, in order to ensure that information is available about all three misalignments. Once the derivatives of the misalignments about each axis with respect to crossing-time are known, crossing-time measurements for several stars can be combined in a least-squares fashion using a sequential estimation technique ${ }^{9,10}$ to arrive at separate estimates of the alignment error for each axis.

The star field that can be viewed by HRDI is restricted by the limb of the Earth in the nadir direction and by the spacecraft in the zenith direction. This restricted field of view limits the viewing of stars whose crossing-time errors are highly dependent on misalignments about the yaw axis. Stars that provide yaw-axis misalignment information cross the telescope field of view from side to side, rather than from top to bottom. Stars near the direction of the orbit normal could be used in this fashion, but they suffer from the fact that their apparent motion (as the spacecraft orbits the Earth) is small, and thus they do not move through the telescope field of view very rapidly. Originally, a series of star-crossing measurements made in a raster-scan fashion was used to determine the yaw misalignment. For each of the series of stars, a slightly different telescope yaw angle, offset from the expected yaw angle of the target star, was used; the entire angular range in which the yaw misalignment was known to fall was covered during the series (see Fig. 3 for an example of the raster-scan yaw method). Since the telescope field of view effectively limits which angle offsets can be viewed (and which target stars are seen), the yaw misalignment can be determined from the average angle at which target stars were actually observed. The yaw misalignment results prior to April 4, 1993, presented in this paper were obtained using this method.

Since April 4, 1993, a different method has been adopted, which combines observations of the normal roll-pitch stars with measurements from several other stars near the orbit-normal direction, to add information about the yaw alignment to the data set. This new method allows a simpler operational procedure (using only one alignment mode) while providing a better result. The disadvantages noted earlier regarding the use of these stars are overcome by carefully selecting the yaw target stars in a narrow ring around the orbit-normal direction, close enough to contribute yaw alignment information, but far enough from the orbit normal to move through the telescope field of view in a short time. (Stars with an azimuth within $15 \mathrm{deg}$ of the pitch axis and an elevation of 5 to 20 deg away from the roll-pitch plane seem to be ideal for this purpose for HRDI.) The HRDI telescope field of view is narrowed to $0.1 \mathrm{deg}$ wide by $0.1 \mathrm{deg}$ long to simplify the data collection and analysis of these yaw target stars.

All of the HRDI alignment measurements are made only during the night portion of an orbit, when the subsatellite point is on the Earth's unlit hemisphere, viewing stars towards the cold side of the spacecraft (away from the sun). These viewing conditions are imposed as safeguards for the instrument, which should neither be pointed at the sun nor see sunlight reflected from spacecraft surfaces, and have the effect of limiting the number of stars in each set of measurements. The actual number of stars used varies from about 20 to 40 , depending on the length of the dark part of the orbit. This range produces adequate results, since the standard deviations of the measured misalignments are now much less than the precision with which the position of the telescope is read (0.0025 deg).

\section{Results}

Figure 4 shows the determinations of the roll, pitch, and yaw misalignments between the HRDI and spacecraft frames, as a function of the number of days since launch (also known as UARS daysUARS was launched on September 12, 1991). Each of the filled dots on the plots represents the measurement from a single day's set of stars, with the error bars showing the standard deviation of the measurement or the telescope read precision, whichever is larger. Typical standard deviations of the measurements are $0.0003 \mathrm{deg}$. Each plot also includes, as a bar on the far right-hand side, a representation of the accuracy required to meet HRDI's wind and position error budget.
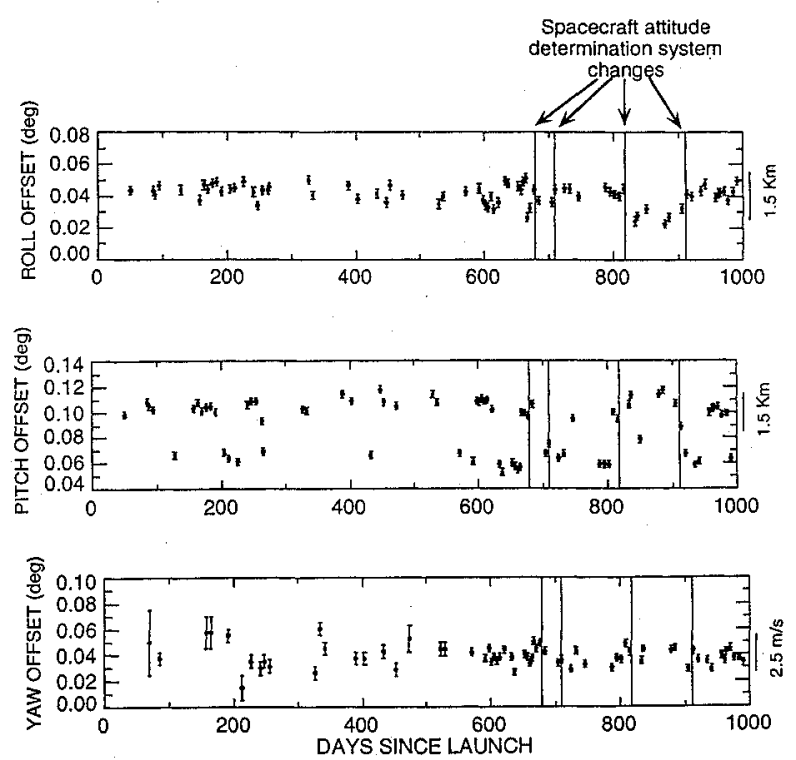

Fig. 4 Measured misalignments between HRDI and UARS. 

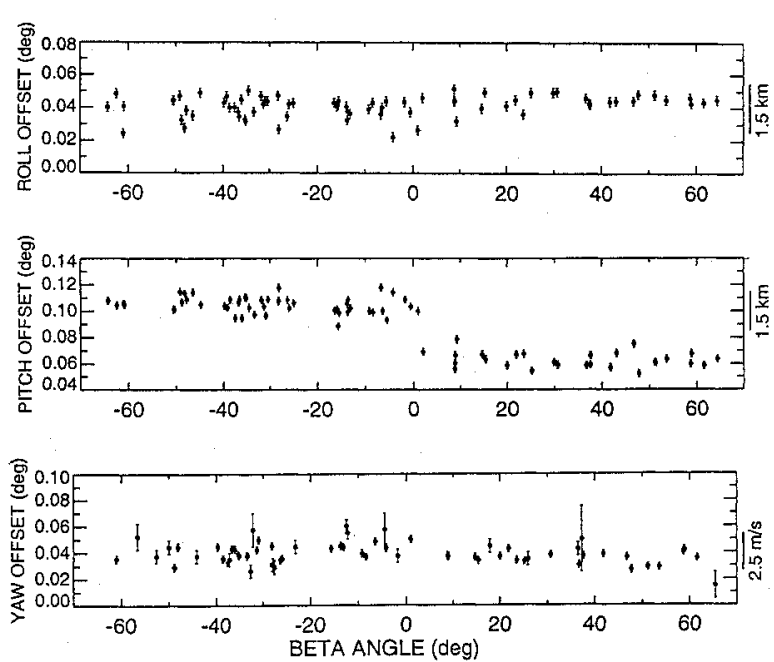

Fig. 5 Measured misalignments between HRDI and UARS as a function of solar $\beta$ angle.

The first item of note is that there are apparently two states in the measurements for the pitch misalignment. Figure 5 shows the same measurements organized by the angle between the spacecraft orbit plane and the sun (termed the solar $\beta$ angle), and demonstrates that the pitch misalignment depends on the sign of this angle. It is believed that this alignment difference is due to the fact that the spacecraft itself is yawed around $180 \mathrm{deg}$ when the solar $\beta$ angle changes sign, to keep the sun on the same side of the spacecraft (toward the solar panel and away from the instruments' radiators). These two states of the spacecraft-sun-Earth alignment may cause differences in the way the spacecraft structure is heated, possibly changing its shape. Since the HRDI instrument is mounted far from the UARS stellar tracking sensors, which are used to determine the spacecraft attitude, the alignment between the spacecraft and the HRDI instrument may be changed.

Closer examination of the roll offsets (for example) plotted in Fig. 4 also shows some periods of larger variation of the measured values from their mean-for example, the period of UARS days 830 through 900 . This apparent noise in the HRDI alignment measurements is actually caused by minor adjustments in the spacecraft stellar tracking software. These adjustments, marked on the plot by vertical lines at the UARS days on which they occurred, are made to enhance the accuracy of the UARS attitude, but since they cause the reported spacecraft attitude to vary slightly for a given real attitude, they cannot be differentiated from changes in the HRDI-UARS misalignment. The adjustments shown are the largest that were made, and were from 10 to $100^{\prime \prime}(0.0028$ to $0.028 \mathrm{deg})$ in magnitude.

Whatever the cause of these misalignment variations, the measurements can be corrected using the known misalignment for the particular state of the spacecraft during the time in question to yield altitudes and velocities that are within the required accuracies. If there is some time constant involved in the step between the two pitch alignment states, it is less than 2 days, which is the minimum time between HRDI alignment measurements in different yaw states.

Figures 4 and 5 also show that the early yaw alignment measurements are not as accurate as the pitch-roll measurements of the same time frame. These yaw measurements were made using the rasterscan method described earlier, which did not produce very accurate results, for several reasons. The first measurements of this type had to be made on a very coarse grid, because this method depends upon having an approximate knowledge of the result before more precise measurements can be performed. After this initial determination was made, it was possible to improve results by concentrating observations near the edges of the raster. Still, this method required several stars to make a single measurement, and thus depended upon all the observations being available, placing particular emphasis on the stars observed very near to the edge of the telescope field of view (where undesirable instrumental effects are maximized). Because not all of the target stars are detected and because there is a limited amount of time available for making these observations, the accuracy of the yaw alignment determination was barely sufficient

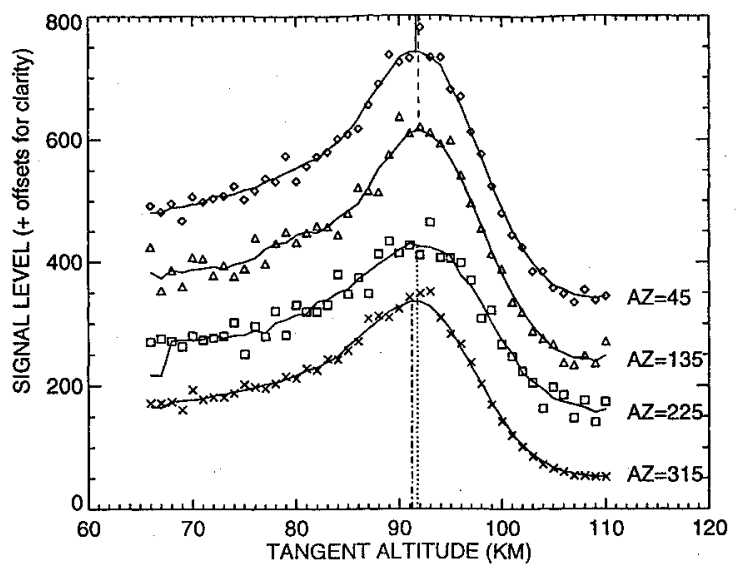

Fig. 6 Measured thin-layer brightness for May 31, 1992, spacecraft flying backward $(A Z=$ azimuth angle, where 0 is along the spacecraft velocity vector).

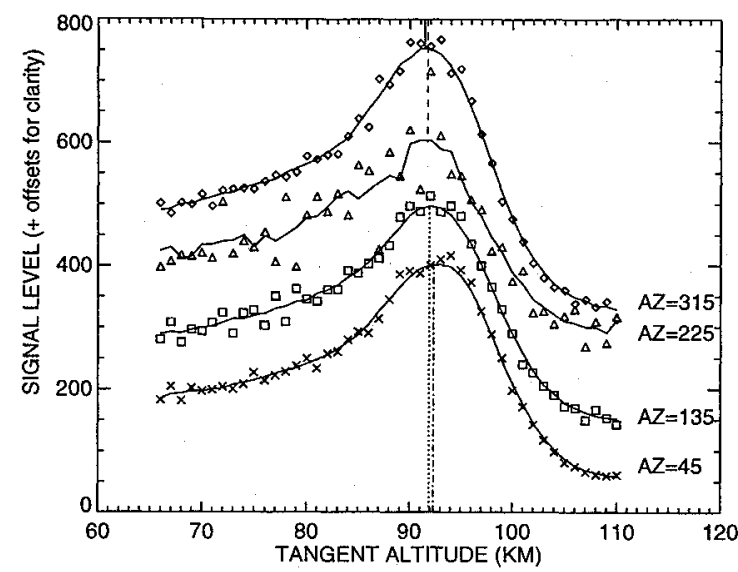

Fig. 7 Measured thin-layer brightness for June 2, 1992, spacecraft flying forward.

to meet the requirements, and it remained a major source of error in the wind results. The adoption of the new combined roll, pitch, and yaw measurement scheme has solved this problem; note that the spread and measurement uncertainty in the yaw offset measurements has improved since UARS day 571 (April 4, 1993), when the new scheme was implemented.

Attempts to verify the measurements of the misalignment have been made in several ways, and in general have confirmed the validity of the technique. One of the most successful of these efforts employed a special instrument measurement sequence, called the four corners mode, in which the instrument viewed the 5577. $O\left({ }^{1} S\right)$ green line emission at night. In this mode, the atmosphere is scanned in altitude from about 65 to $110 \mathrm{~km}$ at each of four azimuths: $45,135,225$, and $315 \mathrm{deg}$. Each scan takes about $30 \mathrm{~s}$, and for these tests the mode was used during an entire 24-h period of nighttime passes (half of each orbit). Because the nighttime green line emission comes from a thin layer near $96 \mathrm{~km}$, the alignment between the instrument and the spacecraft can be verified from the measured brightnesses. Figure 6 shows the averaged brightnesses for all four azimuths at each measurement altitude for May 31, 1992 , during which the spacecraft was flying backwards (negative $\beta$ angle). (For clarity, a different offset was added for each azimuth.) The altitudes at which the brightness peaked, corresponding to the approximate height of the emission layer, are marked with vertical lines. Note that the peak brightness falls between 91 and $92 \mathrm{~km}$ for all four azimuths. Figure 7 shows a similar plot for June 2, 1992, during which the spacecraft was flying "forwards" (positive $\beta$ angle). This figure also shows good agreement for all four azimuths, the peaks falling between 91.5 and $92.5 \mathrm{~km}$. The agreement for the four azimuths in each of these two cases is only possible because the altitudes are adjusted using the known misalignments for the two yaw states. 


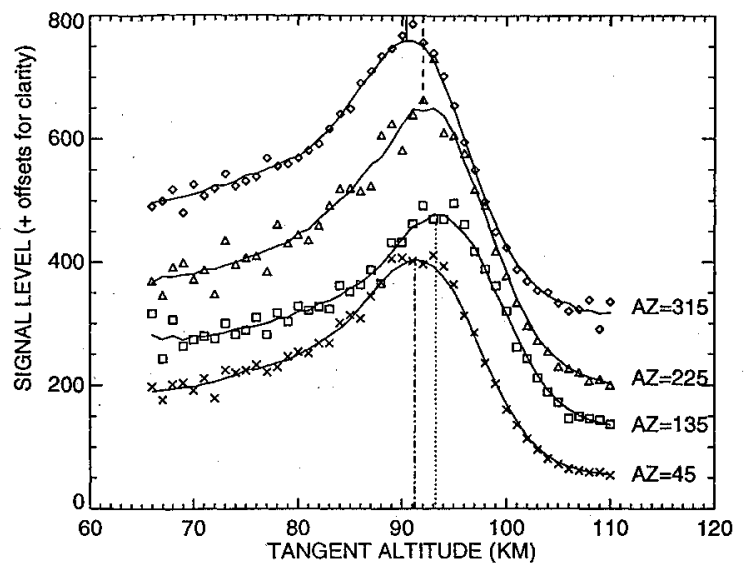

Fig. 8 Measured thin-layer brightness for June 2, 1992, using other (backward) alignment.

Figure 8 shows the brightnesses for each altitude for June 2, 1992, using the alignment adjustments calculated for the backwards yaw state, which is not the correct state for that day. Note the clear offset of about $2 \mathrm{~km}$ between the forward ( 45 and $315 \mathrm{deg}$ ) look directions and their backward counterparts. This result is what would be expected from an offset in the pitch angle of about $0.04 \mathrm{deg}$, which is almost exactly the major difference between the misalignment adjustments being used for the two states. Taken together, Figs. 6-8 provide strong evidence that the misalignments as they are currently characterized are substantially correct-when the calculated alignments are used to adjust the data, the emitting layer is seen at nearly the same altitude for all four azimuths in each spacecraft yaw state, and changing the alignment adjustments creates easily observed offsets of the expected size.

Validation of the alignment measurements using the green line data has an accuracy limitation of about $1 \mathrm{~km}$, or $0.02 \mathrm{deg}$, which is mainly due to changes in the height of the emitting layer over the surface of the earth (which is not sampled in exactly the same way for each azimuth) and the relatively small amount of light given off by the observed layer.

The misalignment measurements have also been verified in another, less direct way. As a part of the HRDI validation effort, HRDI wind measurements have been compared with many measurements taken by radars and rockets. ${ }^{11}$ No discernible systematic altitude offset (which would result from pitch or roll errors) and no systematic velocity offsets (which would result from pitch or yaw errors) have been detected between HRDI and these correlative measurements for either spacecraft yaw state. This comparison might not reveal small errors in the alignment adjustments, but it would certainly show any gross errors on the order of $3-5 \mathrm{~km}$ or $5-10 \mathrm{~m} / \mathrm{s}$.

\section{Summary}

The accuracies of the measurements of the HRDI-UARS misalignments are sufficient to meet the requirements of the HRDI wind measurements. The alignment between the HRDI instrument and the UARS spacecraft has two clearly defined states, depending on the orientation of the spacecraft and the sun, which may be caused by differences in the heating and warping of the spacecraft structure. Minor changes in the measured misalignments can be seen, which result from adjustments in the UARS attitude determination system, but the measured misalignment results otherwise show good longterm stability and also show good agreement with nighttime green line observations. Without this calibration the HRDI data set would be seriously compromised.

\section{Acknowledgments}

This work is sponsored by NASA through Contract NAS 5-27751. We thank Deborah Eddy for her help in preparing the manuscript, and the reviewers for their valuable comments.

\section{References}

${ }^{1}$ Grassl, H. J., Skinner, W. R., Hays, P. B., Gell, D. A., Burrage, M. D., Ortland, D. A., Marshall, A. R., and Abreu, V. J., "Atmospheric Wind Measurements with the High Resolution Doppler Imager," Journal of Spacecraft and Rockets, Vol. 32, No. 1, 1995, pp. 169-176.

${ }^{2}$ Hays, P. B., Abreu, V. J., Dobbs, M. E., Gell, D. A., Grassl, H. J., and Skinner, W. R., "The High-Resolution Doppler Imager on the Upper Atmosphere Research Satellite," Journal of Geophysical Research, Vol. 98, No. D6, 1993, pp. 10,713-10,723.

${ }^{3}$ Reber, C. A., Trevathan, C. E., McNeal, R. J., and Luther, M. R., "The Upper Atmospheric Research Satellite (UARS) Mission," Journal of Geophysical Research, Vol. 98, No. D6, 1993, pp. 10,643-10,647.

${ }^{4}$ Grosch, C. B., "Orientation of a Rigid Torque-Free Body by Use of Star Transits," Journal of Spacecraft, Vol. 4, No. 5, 1967, pp. 562-566.

${ }^{5}$ Mackison, D. L., and Gutshall, R. L., "Star Scanner Attitude Determination for the OSO-7 Spacecraft," Journal of Spacecraft, Vol. 10, No. 4, 1973, pp. 262-267.

${ }^{6}$ Snow, F., Krack, K., Sheu, Y., and Bosl, W., "Accuracy Study of the Upper Atmosphere Research Satellite (UARS) Definitive Attitude Determination," Proceedings, Flight Mechanics/Estimation Theory Symposium (NASA Goddard Space Flight Center, Greenbelt, MD), May 1988, pp. 26-41.

${ }^{7}$ Shuster, M. D., Pitone, D. S., and Bierman, G. J., "Batch Estimation of Spacecraft Sensor Alignments, 1. Relative Alignment Estimation," Journal of the Astronautical Sciences, Vol. 39, No. 4, 1991, pp. 519-546.

${ }^{8}$ Gottlieb, D. M., "SKYMAP: A New Catalog of Stellar Data," Astrophysical Journal Supplement, 38, 1978.

${ }^{9}$ Ortland, D. A., Hays, P. B., Skinner, W. R., Burrage, M. D., Marshall, A. R., and Gell, D. A., "A Sequential Estimation Technique for Recovering Atmospheric Data from Orbiting Satellites," The Upper Mesosphere and Lower Thermosphere, edited by R. M. Johnson and T. L. Killeen, Vol. 87, Geophysical Monograph Series, American Geophysical Union, Washington, DC, 1995.

${ }^{10}$ Rodgers, C. D., "Retrieval of Atmospheric Temperature and Composition from Remote Measurements of Thermal Radiation," Review of Geophysics and Space Physics, Vol. 14, No. 4, 1976, pp. 609-624.

${ }^{11}$ Burrage, M. D., Gell, D. A., Hays, P. B., Marshall, A. R., Ortland, D. A., Skinner, W. R., Franke, S. J., Fritts, D. C., Hoffman, P., Manson, A. H., McLandress, C., Niciejewski, R., Schmidlin, F. J., Shepherd, G. G., Singer, W., Tsuda, T., and Vincent, R. A., "Validation of Mesospheric and Lower Thermospheric Winds from the High Resolution Doppler Imager on UARS," Journal of Geophysical Research (to be published).

A. L. Vampola Associate Editor 